\title{
Research on Digital Power Grid Information Integration Solution
}

\author{
Jiye Wang ${ }^{1}$, Hengzhi Cui ${ }^{2}$, Hanyong Hao ${ }^{3}$, Hongyu $\mathrm{Yao}^{4}$ and Lishan Bao \\ ${ }^{1}$ State Grid Corporation of China, No.66, West Changan Road, Beijing, China \\ ${ }^{2}$ State Grid Jiangsu Electric Power Company, No.215, Shanghai Road, Nanjing, Jiangsu, China \\ ${ }^{3}$ State Grid Corporation of China, No.66, West Changan Road, Beijing, China \\ ${ }^{4}$ YOYO SYSTEMS INC, 3F East Area, YiQing Tower,No. 38 Guangqu Road, ChaoyangDistrict, Beijing, China \\ ${ }^{5}$ State Grid Jiangsu Electric Power Company Information \& Telecommunication Branch, No.20, Beijing West Road, Nanjing, Jiangsu, \\ China
}

\begin{abstract}
Information integration is an important part of the digital grid architecture. The purpose is to solve the information interaction obstacles between heterogeneous systems on the basis of making full use of the old system. This paper analyzes the development stage of digital power grid information integration from the perspective of information integration, and points out that the information integration of current digital power grid is mainly data-oriented integration. Based on the characteristics of digital power grid information integration, this paper puts forward a digital power grid information Integration solution combining horizontal information integration and vertical information integration, designs the overall architecture of digital power grid information integration, and elaborates the horizontal integration and vertical integration respectively.
\end{abstract}

\section{INTRODUCTION}

In different development periods, electric power enterprises have installed and developed application systems to realize different functions according to the specific needs of each business department. Because of the different periods of development of these application systems, different functions and different application providers of that, and also because the application system is concerned about the different aspects of power objects as well as, the power object has its own specialized modeling methods and uses different databases, operating platform, and development language., the data models and information between them are inconsistent. Therefore, information redundancy and information island are difficult to avoid. Each application system becomes a relatively isolated "automation island" in information. They can not exchange data with other application systems or achieve information sharing and integration within the enterprise [1-2]. The inconsistency of data model and the inability of information integration increasingly become the bottleneck restricting the improvement of electric power automation level. There is an urgent need for an integrated information integration solution with high standardization, good openness and effective handling of massive amounts of information.

The title is set in bold 16-point Arial, justified. The first letter of the title should be capitalised with the rest in lower case. You should leave $35 \mathrm{~mm}$ of space above the title and $6 \mathrm{~mm}$ after the title.
At the same time, the information communication of digital power grid has hierarchical characteristics.[3-4] Power grid information is acquired from the power grid through the acquisition device, mainly through the power dispatching data network and the like transmission to the dispatching control center. Power dispatching data network uses power fiber and microwave to establish broadband and High-performance network platform. Mainly transmitting the power production data and managing the information data. Therefore, the information integration of digital power grid should not only solve the problem of information sharing among different application systems, namely horizontal information integration, but also solve the problem of information interconnection among different power control centers and that is the vertical information integration.

Based on the actual needs of the digital power grid information integration, this paper puts forward an information integration solution to effectively solve the information isolated island of the digital power grid. Based on data integration, the scheme integrates the horizontal and vertical information integration strategy and enterprise application integration scheme, which can solve the information integration problem of the digital power grid in an all-around way [5-6].

\footnotetext{
* Corresponding author: lvjerry0930@ outlook.com
} 


\section{DEVELOPMENT STAGE OF DIGITAL POWER GRID INFORMATION INTEGR- -ATION}

The theory of information integration points out that with the continuous evolution of integration requirements and the increasing complexity of requirements and process, information integration presents distinctive stage features. The development track is as follows:

\subsection{Data Transmission}

The initial integration requirements generally start from the data transfer that is to say there is a need to transfer some data from the system at site A to the system at site B. In general, the techniques adopted were through a stage of the network protocol transmission, disk transmission, file transfer, programming and development of communication transmission program, using database tools for replication, the use of messaging middleware for transmission, the use of data warehouse data extraction tools for transmission and other processes [7-8].

\subsection{Data Integration}

After a large amount of data was generated, new problems emerge and new demands are put forward: Because different application data formats or caliber is different, it is hoped that the computer can complete translation conversion automatically; Hope to be more convenient to complete the data aggregation and distribution; Hope to be able to share another system data in real time; Hope to provide more convenient data synchronization mechanism and so on. Technically, people achieve their goals through FTP, programming development, database gateways, messaging middleware technology, data warehouse technology, ODBC, JDBC and other forms [9-10].

\subsection{Application Integration}

The existence of discrete business applications makes the chain of business management disjointed. Therefore, people want to realize the tightness of business monitoring through the function cohesion of crosssystem. With the integration requirements going deeper, a lot of business operations are involved in a number of application systems. How to manage and monitor the "electronic process" collaboration among multiple applications in a convenient and easy-to-maintain way becomes a matter of concern. Technically, people realize cross system function calls through open API, adapter, workflow tools and other technical forms [11-12].

\subsection{Service-oriented Integration}

Through WEB SERVICE and other standardized service component protocols/specifications, people can get whatever service they need at any time and place, without having to care about the technical implementation of the service.

According to the development status of digital power grid, this paper considers that the information integration of digital power grid is in the stage of data integration and Application Integration. Figure 1 shows the various stages of data integration and the location of digital power grid information integration.

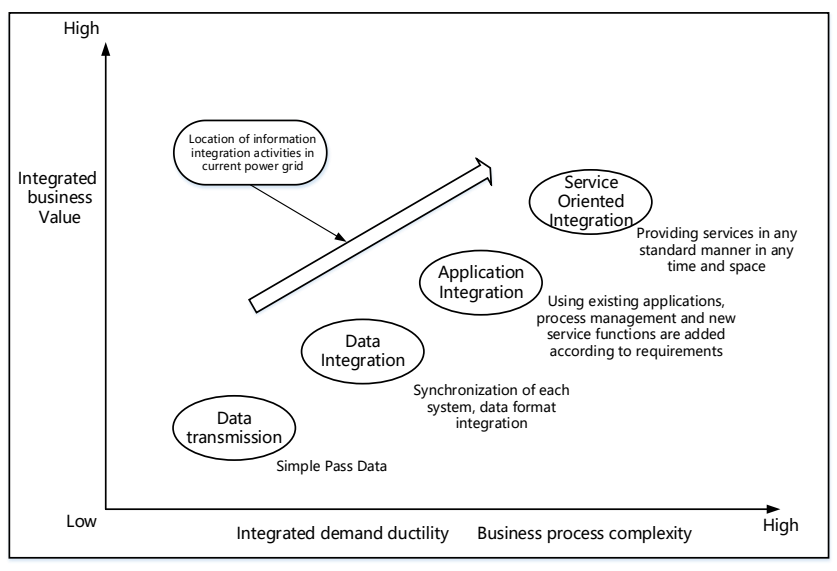

Fig.1. Development stage of information integration

\section{DIGITAL POWER GRID INFORMATION INTEGRATION OVERALL STRUCTURE}

According to the actual demand of digital power grid information integration, the overall architecture of digital grid information integration proposed in this section considers the implementation of horizontal integration and vertical interconnection respectively. Horizontal integration is the rational organization of unified model data in a unified Department, building a data center for advanced applications to analyze data, information platform across the physical separation device Depending on the size of the total amount of information on both sides of the building under separate construction(to image transfer the common information) or unified construction (to ensure information security to join the isolation link); Vertical integration is for the upper and lower scheduling center through the power 
communication network to achieve information integration, efficiently splicing and splitting of the grid model to provide appropriate data to advanced application modules [13-14]. Figure 2 presents the structure diagram of the digital power grid information integration framework.

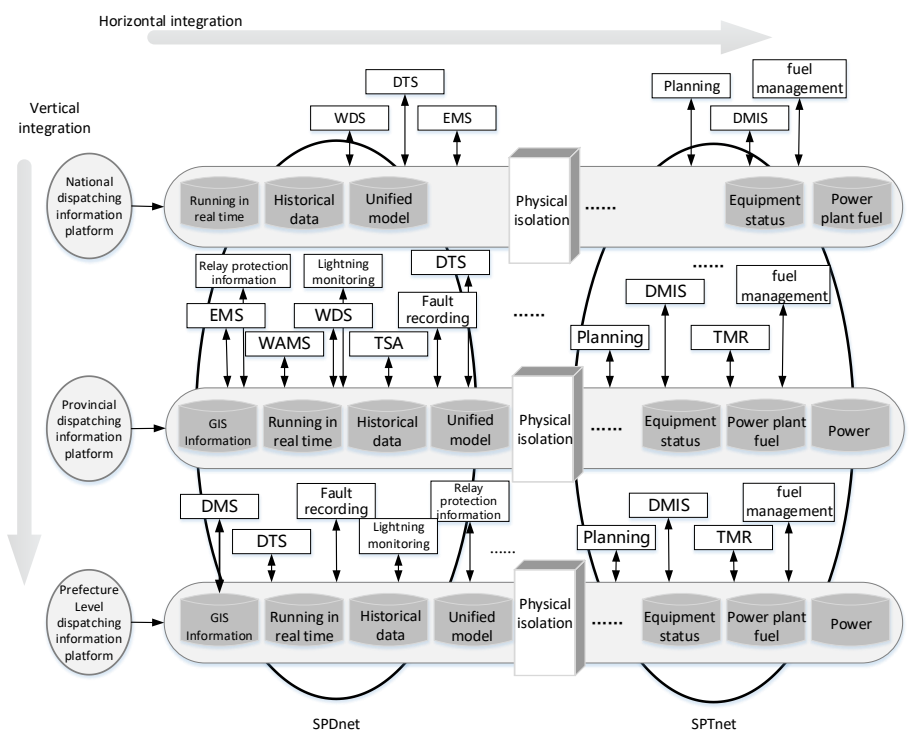

Fig.2. Overall framework of information integration in digital power grid

\section{INTEGRATED DESIGN OF DIGITAL POWER GRID APPLICATION}

\subsection{The important feature of the digital power grid is the integration of power grid monitoring and production operation management}

Enterprise application integration (EAI) is an important content of information integration of digital power grid.

The requirement of enterprise application integration is changing and enriched constantly.

From the integration of function to the integration of process, the integration from the internal application of enterprise to the integration of across enterprise boundary.

\subsection{The purpose of EAl is to centralize enterprise information system resources}

The EAI solution is also in constant development, from early point to point integration to hub based integration (Hub integration), and now it's based on Enterprise Service Bus (ESB) integration [15-16], as shown in Figure 3.

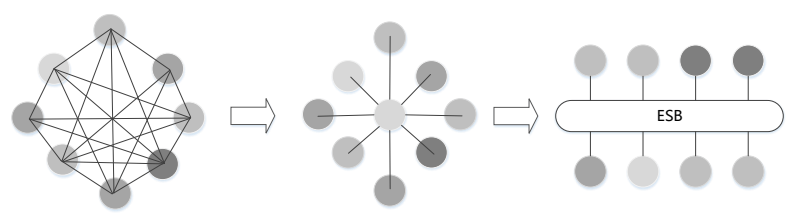

Fig.3. Evolution process of enterprise application integration

\subsection{Application integration consists of the following levels:}

Point to point application interface level integration: Such methods are through the application interface to achieve the application system integration, to provide an almost real-time integration. However, the interface development of the call is one to one, which is not conducive to the development of large-scale systems. At the same time, the logical order of function calls cannot be flexibly modified.

Hub business process integration: The implementation of business process integration is usually accomplished by using some high-level Middleware. In this scheme, the service agent can only manage function calls for different applications, not the whole business process execution. At the same time, the implementation of agent layer has high requirements for development technology, and different middleware technologies are technically limited.

The above two methods are the integration of nonfunctional layer, which belongs to tightly coupled application system integration mode. This tightly coupled integration mode will affect the flexibility and expansibility of the system, hinder the business process adjustment and optimization, and is not conducive to the business development of enterprises. Based on serviceoriented architecture (SOA) for enterprise application integration it provided a business-oriented business services layer bus-based integration model. This model not only guarantees the original system data security and logical security but also can realize the loose coupling among the systems and facilitate the restructuring and optimization of the business process system.

Service Oriented Architecture (SOA) is an application architecture for all functions of digital grid information system, which is precisely defined, invoked, independent service, and can be arranged orderly to construct the application architecture of business process. The typical features of SOA are that it's independent of 
platform, loosely coupled and it has oarse-grained

In a service-oriented architecture, there are mainly three types roles: service requester, service provider and service registration center. There are three main operations among roles: publishing, searching and binding, as shown in Figure 4.

Concrete implementation of service provision by the service provider: The service requester obtains from the service registry the structure of the message it must be sent and received from the service provider and the protocol used to be accessible to the service. The service requester does not need to know the internal implementation way of the service provider.

SOA based enterprises can effectively reorganize the system function modules according to the needs of business growth, encapsulate and reuse legacy systems to form new services, develop new systems according to service specifications, establish new services, and at the same time guarantee the loose coupling among services to the maximum extent.

SOA is a new idea in the field of software engineering. It is a new model of software development, that is, by building service integration business information needs of the system, to show the internal application. No matter it is a legacy application system, or it is a new application system, it can be packaged in accordance with the specification of standard services, hiding application internal implementation details. As a result, the automated systems with different functions or provided by different vendors can be easily integrated within the enterprise's service-oriented unified framework through standard service encapsulation, Centrally managed by the service registry, releasing services to the outside. So it can be easily invoked by the service requester, realizing the sharing of data and the circulation of information in the whole enterprise.

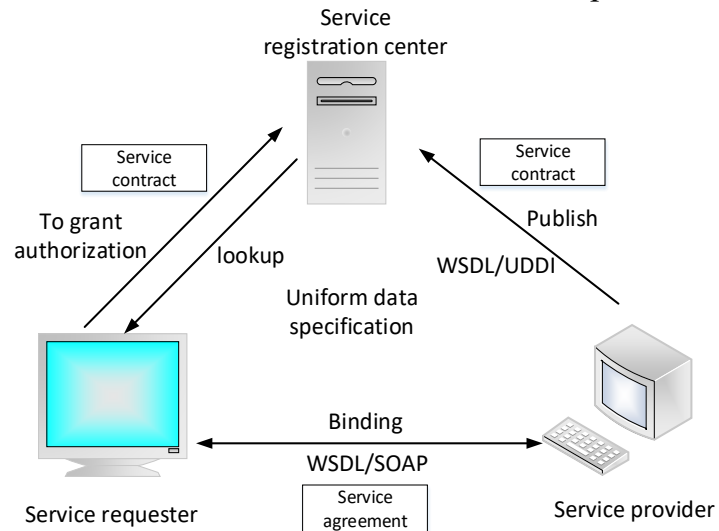

Fig.4. SOA model

\subsection{Design of application integration platform based on SOA}

service, good encapsulation and flexibility [17-18].

Traditionally, a system that enables enterprises to execute interprocess communication is called Middleware. Middleware is primarily concerned with data layer integration, which means that these systems do not provide any functionality that can be used for higher-level integration. On the contrary, the enterprise application integration (EAI) includes the technical level and process definition level, enabling a number of customized and/or encapsulated business applications to exchange semantic layer information in a mutually understandable format and context. That is, EAI products not only integrate applications at the technology level but also provide a communication framework. The framework can support the integration of information systems in the integration of semantic layer (object-oriented level) and pragmatic layer (pay attention to process level integration)[19-20].The EAI platform based on SOA should provide the following services, as shown in Figure 5.

(1) Connection service: a service that extracts information from an application and then passes it to another application. It includes communication services, addressing services and security services, which can realize data layer integration.

(2) Interface services: services provided for customized and encapsulated applications to communicate with other systems through different interfaces provided by them. It includes interface conversion service and metadata expression service.

(3) Transfer service: the core of the EAI system, which makes the object can be integrated through the acquisition of the message conveyed by the connection service and converting it to a destination acceptable format. It includes identification service, synchronization service and routing service.

(4) Process Management Services: Integrating processes within and across businesses becomes possible by operating at the level of individual transitions. The process management service controls the execution of the transformation order specified by a pre-defined process model. With process management services, multiple distributed applications can be integrated to obtain new enterprise applications.

(5) Runtime service: Including distributed services, upgrade services and monitoring services.

(6) Development Service: In order to support the development of new adapters, transformation models and process models, development services include interface development services, transformation specification services, and process modeling services. 


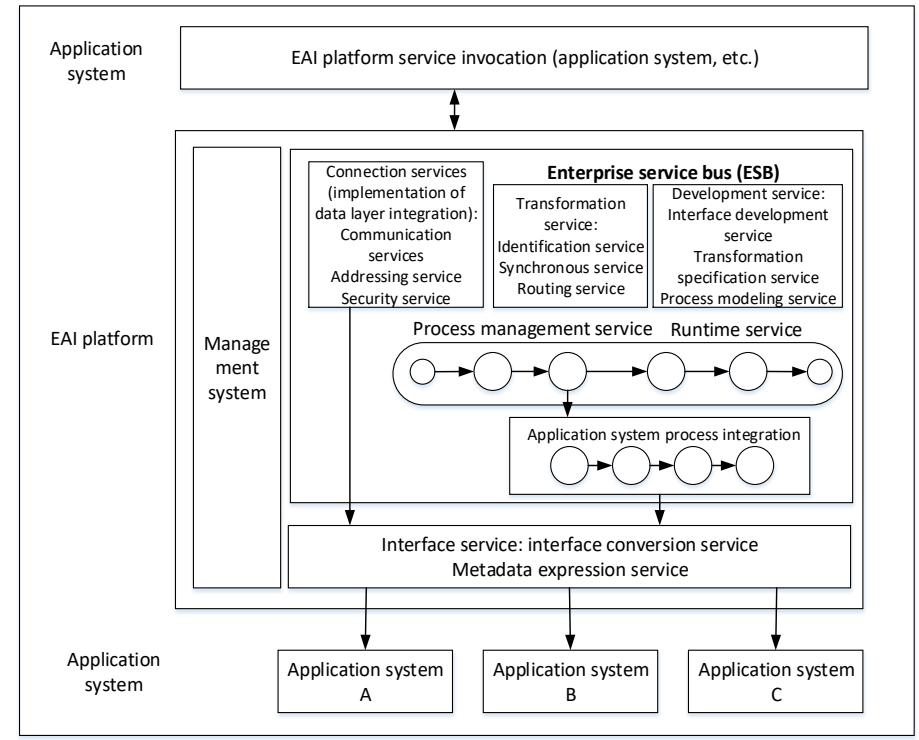

Fig.5. EAI platform based on SOA

\subsection{Business Process Integration Based on SOA Integrated Platform}

Different from traditional EAI, SOA based business process integration can successfully create models, automate process processing, and monitor and manage these business processes, to meet the needs of business change [21-22].Figure 6 is a business process integration diagram based on SOA.

The core of SOA is service, Figure 6 shows the different levels of service in an enterprise application system. According to the characteristics of enterprise business, in the enterprise service environment, service granularity is different. The services in the diagram are divided into the business process layer, the service component layer, and the Atomic service layer. Business process layer is the top level service of enterprise, and it's the application layer of direct human-computer interaction. The application of business process layer is the application system of enterprise operators for daily operation and daily affairs, combining business process choreography definition, calling different service components to complete.

The definition of business process layer is completed by building process definition document. And the components that build the process definition are provided by the service component layer and the atomic service layer.

The service component layer is the object that business process administrators need to manage. According to the basic business requirements of the enterprise, this layer defines the basic services. Of course, these services are composed of atomic services in accordance with the certain business logic.

The atomic service layer is the basic service layer that directly encapsulates different functions of the application system. According to the characteristics of the application system and the development interface of the application system, this layer has developed the services to realize different functions. These different services use the service registry for unified management and can be self-describing through service contract files. The service component layer can invoke these atomic services according to the needs of the application to realize the basic business activities of the enterprise.

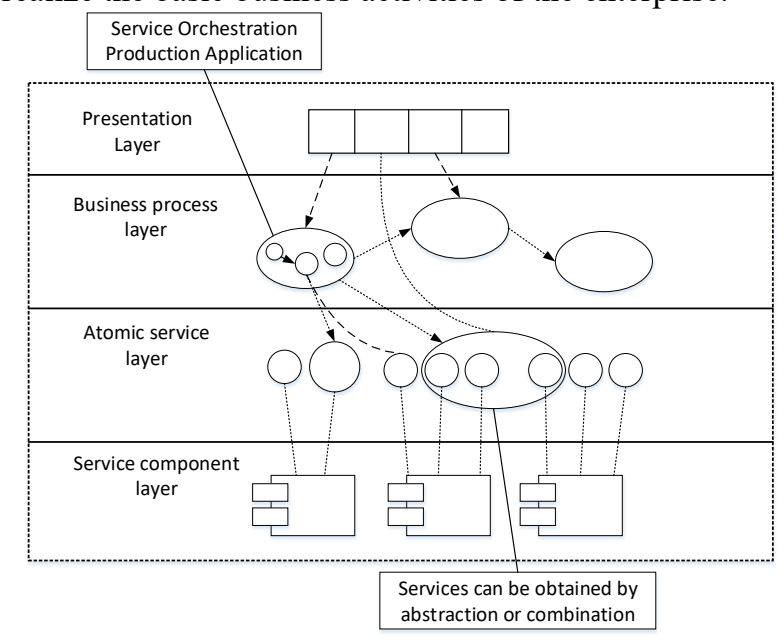

Fig.6. Business process integration based on SOA

\section{CONCLUSION}

This paper first describes the importance of information integration in the realization of the digital power grid. It points out that information integration is an effective way to solve the problem of "information isolated island". It analyzes the digital power grid information integration stage of development, and points out that the current digital grid information integration is mainly oriented to the integration of data. It analyzes the characteristics of digital power grid information integration and pointed out that the digital grid information integration should include horizontal information integration and vertical information integration. This paper puts forward an information integration solution which can effectively solve the information isolated island of the digital power grid. Based on data integration, the scheme integrates the information integration strategy of two directions in horizontal and vertical direction, which can solve the 
information integration problem of the digital power grid in an all-around way.

\section{REFERENCES}

1. Yang Z, Li J, Li M, et al. Application Analysis of Power Grid Dispatching Automation System[J]. Electrical Engineering, 2016.

2. Hauswirth A. Applications of AC Optimal Power Flow in Distribution Networks[J]. Australian Educational Researcher, 2015, 26(2):45-53.

3. Rahman N, Rutz D, Sugumaran V. Building Data Warehouses Using Automation[J]. International Journal of Intelligent Information Technologies, 2015, 11(2):1-22.

4. Lu Guang, Zhang Boming, Sun Hongbin. Application of data warehouse and data mining technology in power system [J]. power grid technology, 2001, 25(8): 54-57.

5. Zheng Yi. Metadata model in the application of data integration platform [D]. North China Electric Power University, 2007.

6. Huang Hongbin, Deng Su, Ma Lili, Zhang Weiming, Liu Zhen. Semantic Oriented metadata model and OWL representation [J]. computer engineering and science, 2007, 29(11): 105-109.

7. Wang Shaofeng. Object oriented technology UML tutorial $[\mathrm{M}]$. Beijing: Tsinghua University press, 2004.

8. Liu Qingrui, Zhang Qiang.IEC61970 several major software technology applied in [J]. power automation equipment, 2006, 26(6): 89-92.

9. Tian D. Study on automation technology of electric equipment in power plant[J]. Heilongjiang Science, 2017.

10. Extension of.IEC61970 standard, such as Sun Hongbin, Wu Wenquan and Zhang Boming, in the integration of dispatching center $[\mathrm{J}]$. power grid technology, 2005,29(16): 21-25.

11. Luo Q, Liangliang W U, Shou T, et al. Decision support system for power grid planning and management based on CIM[J]. Power System Protection and Control, 2016.

12. Milovanovic V, Milicev D. An interactive tool for UML class model evolution in database applications[M]. Springer-Verlag New York, Inc. 2015.

13. Zhu Ling, Xue he. Mapping model of object relational database system and its application [J]. computer science and engineering, 2007, 29(12): 120-122.

14. Liu Bo. Research on several problems of dispatching data sharing platform based on security partition and IEC61970 [D]. Zhejiang University, 2006: 9-12.

15. David Linthicum. Enterprise Application Integration. Addison-Wesley Professional, 1999.
16. Gorkhali A, Li D X. Enterprise Application Integration in Industrial Integration: A Literature Review[J]. Journal of Industrial Integration \& Management, 2017:1650014.

17. Zaslavsky I. Service-Oriented Architecture[J]. 2016.

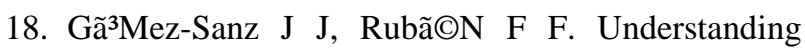
Agent-Oriented Software Engineering methodologies[J]. Knowledge Engineering Review, 2015, 30(4):375-393.

19. Lu Guang, Boming Zhang, Hong-bin Sun. Application of data warehouse and data mining technology in power system [J]. power grid technology, 2001,25(8): 54-57.

20. Farias T M D, Roxin A, Nicolle C. IfcWoD, Semantically Adapting IFC Model Relations into OWL Properties[J]. 2015.

21. Shen Xiling, Ren-yi Liu, Nan Liu. Design and implementation of a work engine based on relational data model [J]. Journal of Jiangnan University (NATURAL SCIENCE EDITION), 2007,6(5): 537541.

22. Pai $\mathrm{T}$ K A. A Study On Power System Automation[J]. 2015, 4:7605-7610. 